\title{
Frequency effects in the abstraction of linguistic ideas
}

\author{
MARILYN D. WANG \\ University of Maryland Baltimore County, Baltimore, Maryland 21228
}

\begin{abstract}
The linear linguistic integration effect reported by Bransford and Franks (1971) has been widely accepted as a demonstration of the ability of subjects to abstract and integrate information from isolated sentences. In two experiments a simple frequency model was successfully used to predict mean sentence recognition ratings obtained with the Bransford-Franks paradigm. Since the simple frequency model does not assume an integrated representation in memory, it is suggested that the linear effect reflects decision processes occurring during the recognition task rather than encoding in memory.
\end{abstract}

In a well-known study, Bransford and Franks (1971) reported data demonstrating a linguistic integration effect in sentence memory. Listeners were presented with several nonconsecutive sentences; each was derived from a single complex idea, but none completely expressed that idea. The subjects were later unable to distinguish sentences actually presented from new sentences also derived from the complex idea. Moreover, as the complexity of the recognition sentence increased, that is, as it more completely expressed the complex idea, recognition confidence ratings increased linearly.

In interpreting their findings, Bransford and Franks argued that the subjects had not stored separate memory representations for each stimulus sentence but, rather, had integrated the ideas presented in the individual sentences to form a single holistic representation of the complete idea. This would, of course, account for the subjects' inability to discriminate old from new sentences. It would also explain why, of all recognition sentences, the one most likely to be "recognized"and recognized with the highest confidence was always the new sentence which expressed the entire complex idea. The basic findings of this study have been replicated and extended by other investigators (Cofer, 1973; Franks \& Bransford, 1972; Griggs, 1974; Katz, 1973; Singer, 1973; Singer \& Rosenberg, 1973).

Results of other studies suggest, however, that the linear recognition effect does not result from semantic processing of information during acquisition. The effect has been found with sequences of related and unrelated words (Small, 1975), syntactically structured sequences

This study was supported in part by a faculty research fellowship awarded by the University of Maryland Baltimore County. Computer time was provided by the Computer Science Center of the University of Maryland. The author wishes to thank Cheryl Berryman, Lisa Gemma, and Robert Ciulla for assistance in collecting the data. Requests for reprints should be sent to the author at: Department of Psychology, University of Maryland Baltimore County, Baltimore, Maryland 21228. of nonsense syllables (Katz \& Gruenwald, 1974), letter and digit sequences (Reitman \& Bower, 1973), and visual patterns (Franks \& Bransford, 1971; Neumann, 1974). Katz (1973) has shown that the effect disappears when recognition instructions are modified and subjects are only required to judge whether recognition sentences have the same meaning as acquisition sentences. Katz, Atkeson, and Lee (1974) have shown that sentences consisting of a single idea are rated much higher when the acquisition list consists entirely of single-idea sentences, and they suggest, therefore, that the linear effect is an artifact of the method of presenting sentences of varying complexity during acquisition. These studies, taken together, suggest that the linear "linguistic" effect is a function of decision processes occurring during recognition rather than a function of semantic integration occurring during acquisition.

Both Neumann (1974) and Reitman and Bower (1973) have pointed out that the Bransford-Franks paradigm is essentially a concept-formation task in which subjects are tested for recognition of the specific instances of a concept which were presented during acquisition. Although neither of these studies used linguistic stimuli, both demonstrated that a linear recognition function can be accounted for by a recognition model in which the recognition rating for a given item is a function of the frequency of presentation of its elements during acquisition, provided the recognition item contains no new elements.

According to a simple frequency model, in the typical linguistic integration experiment, the subject stores separate representations for each single idea, for each pair of ideas, and for each triplet of ideas presented. Associated with each representation is a simple frequency count, and recognition ratings are assumed to be some function of these frequencies. Such a model accounts for the linear effect, since the longer, more complex sentences, in general, have higher frequency counts associated with them. It can also account for the inability of subjects to discriminate old from new 
sentences, provided the frequency measures are comparable for old and new sentences of the same complexity.

Since neither of the studies in which frequency models have been proposed used sentences as stimuli, it is possible that such models are appropriate only when the stimuli are nonlinguistic and that they are not applicable when words or sentences are used as stimuli. Two things suggest that this is not the case. First, frequency effects have been obtained in word-recognition studies (Underwood, 1972). Underwood and Zimmerman (1973) found that a single previous exposure to the syllables of a recognition word was sufficient to increase false recognition responses. In their study false recognition of a word like insult was greater when words like instruct and consult had appeared in the acquisition list. Second, data reported by Bransford and Franks (1971) are consistent with a frequency model. For one of the complex ideas (The ants in the kitchen ate the sweet jelly which was on the table) a frequency analysis reveals that more complex sentences do have higher frequency counts. In addition, with sentence complexity held constant, frequency is an excellent predictor of recognition ratings.

Since frequency information is not available for the other complex ideas used by Bransford and Franks (1971), the present study was conducted to determine how well a simple frequency model can account for recognition ratings obtained with the Bransford-Franks paradigm.

\section{EXPERIMENT 1}

\section{Method}

Subjects. Twenty-one subjects were recruited from an introductory psychology class at the University of Maryland Baltimore County. The subjects received course credit for their participation in the experiment.

Materials. Following Bransford and Franks (1971), acquisition sentences were generated from four complex ideas: The rock which rolled down the mountain crushed the tiny hut at the edge of the sea. The barking dog chased the brown cat which jumped on the girl, The ants in the kitchen ate the sweet jelly which was on the table, and The thunder which was crashing through the valley shook the tinkling bells in the corner. For each complex idea, the acquisition list consisted of two sentences containing one simple idea, two sentences containing two simple ideas, and two sentences containing three simple ideas. Also included were four filler sentences containing one, two, three, and four simple ideas, respectively. The resulting 28 sentences were randomly ordered, with the restriction that no two sentences from the same complex idea be presented consecutively.

The recognition sentences consisted of three old sentences from each complex idea containing one, two, and three simple ideas, respectively, and four new sentences containing one, two, three, and four ideas. Also included were the four old filler sentences and four new filler sentences with one, two, three, and four simple ideas. The order of the recognition sentences was randomized under the same restriction used with acquisition sentences.

A set of cards, each containing four squares of colored paper, was used in a color naming task which was given during the acquisition phase of the study.
Procedure. The subjects were tested in a single group. They were told that their task involved short-term memory for information in sentences. The experimenter read a sentence from the acquisition list and then displayed one of the cards with four colored squares. The subjects, as a group, read the names of the colors aloud. The experimenter then read a short elliptical question concerning the previous sentence, and the subjects wrote the answer to the question. The intertrial interval was about $5 \mathrm{sec}$. Two practice sentences were given before the acquisition sentences were presented.

There was a brief rest period of about $3 \mathrm{~min}$ following the acquisition task. The subjects were then informed that they would be read a list of sentences, some of which were identical to those presented during the first part of the experiment and some of which were very similar, but not identical, to those heard earlier. For each sentence the subjects were required to make a judgment of "old" or "new" and were asked to rate their confidence in the correctness of their choice on a 6-point scale ranging from 0 (no confidence at all) to 5 (very high confidence). The intertrial interval for the recognition task was also about 5 sec.

\section{Results}

Recognition responses were converted to a rating scale ranging from -5 , for high confidence that the sentence was new, to +5 , for high confidence that the sentence was old. In a few instances subjects failed to give a confidence rating, and the response was scored as " 0 ." Mean ratings for old sentences containing one, two, and three simple ideas were $1.52,2.54$, and 3.43 , respectively. For new sentences containing one, two, three, and four ideas, the mean ratings were $.42,2.26$, 2.24 , and 3.59.

In order to determine whether mean sentence ratings varied as a function of sentence type, a repeated measures analysis of variance was performed, excluding the filler sentences. The sentence-type factor contained seven levels for the three types of old sentences and the four types of new sentences. There was a highly significant main effect due to sentence type $[F(6,120)=$ 11.13]. (For significant effects, $p<.001$ unless noted otherwise; for nonsignificant effects, $p>.05$.) There was also a significant linear trend within old sentences $[F(1,20)=21.56]$ and within new sentences $[F(1,20)=$ $31.90]$. Although the old sentences tended to receive higher ratings than the new sentences of the same complexity, the difference was not significant $[F(1,20)=$ 1.73]. These results replicate once again the basic findings of Bransford and Franks (1971), that is, the inability of subjects to discriminate between old and new sentences, and the linear trend in recognition ratings as a function of the number of simple ideas in the recognition sentence.

In order to determine whether a simple frequency model could account for the linear trend in recognition ratings, three frequency counts $\left(F_{1}, F_{2}\right.$, and $\left.F_{3}\right)$ were calculated for each recognition sentence, and a multipleregression analysis was performed using the mean recognition rating for each sentence as the dependent variable. $F_{1}, F_{2}$, and $F_{3}$ were the cumulative frequencies of the simple ideas, pairs of simple ideas, and triplets of simple ideas, respectively. $F_{1}, F_{2}$, and $F_{3}$ were entered 
into the regression equation in that order. $F_{1}$ contributed significantly to the prediction of recognition ratings $[\mathrm{r}=.612, \mathrm{~F}(1,26)=15.61]$. The increments due to $F_{2}$ and $F_{3}$ were not significant $[F(1,25)=1.45$ and $\mathrm{F}(1,24)=2.33$, respectively $]$.

\section{EXPERIMENT 2}

The second experiment was essentially a replication of Experiment 1 with some procedural changes. Two sets of acquisition sentences were administered to different groups of subjects, and the two sets were then combined to form a single recognition list for all subjects. This makes it possible to determine whether the frequency model predicts performance equally well for the same sentences when they are old and when they are new. The second major change concerned the syntactic structure of the sentences. In Experiment 2 the simple ideas were combined in conjunctive sentences rather than in the usual embedded form. This permitted presentation of certain combinations of simple ideas which could not be presented in embedded form, for example, The ants were in the kitchen and the jelly was sweet. However, since these changes also make the number of ideas in each acquisition sentence more salient, and since this has been shown to affect recognition ratings for sentences containing four simple ideas (Small, 1975), two of the complete complex ideas were included in each acquisition list.

\section{Method}

Subjects. Fifty-two subjects were recruited from an introductory psychology class at the University of Maryland Baltimore County and received course credit for their participation.

Materials. Two sets of acquisition sentences were prepared. Sentences were based on the same complex ideas as in Experiment 1. For each complex idea, two sentences containing one, two, and three ideas, respectively, were assigned to each acquisition set. Two complete complex ideas were included in each acquisition set and each was presented twice. The 28 acquisition sentences in each set were randomly ordered, as in Experiment 1, and the order was matched for the two sets as closely as possible.

For the recognition task, the acquisition sentences from both sets were combined to form a 52 -item recognition list. Items were randomly ordered as above.

Procedure. The subjects were tested in four groups. Two groups received each set of acquisition sentences. For one group in each acquisition condition, the recognition list was presented in the original order, and for the other group the order was reversed. Task instructions to the subjects were the same as in Experiment 1 except that the intervening task was changed. Immediately following the acquisition sentence, the experimenter read a number and the subjects were required to count backward by threes, filling in the numbers on their response sheets. After about $5 \mathrm{sec}$, the elliptical question was read. Procedures for the recognition task were the same as in Experiment 1.

\section{Results}

A mixed-design analysis of variance was performed on the recognition ratings, with acquisition set and recog- nition list as the between-subjects factors and recognition sentences as the within-subjects factor. The recognition sentences were nested in two sets corresponding to the two sets of acquisition sentences. The effect of sentences was highly significant $[F(50,2600)=9.38]$. There was an interaction of Acquisition Set by Recognition Sentence Set $[F(1,52)=23.62]$. This interaction is equivalent to a main effect for old vs new sentences. Both acquisition set and recognition list interacted with sentences $[F(50,2600)=1.32, p=.05$, and $F(50,2600)=$ 2.28 ], although neither interaction was readily interpretable. Other investigators have noted certain systematic changes in recognition ratings during the course of a recognition list (Cofer, 1973), and such effects could be responsible for the observed interactions since the acquisition set groups differed with respect to which sentences were old, and the recognition list groups differed in the order of presentation of the recognition sentences.

Of primary interest was the mean for each type of sentence when it was old and when it was new. Means for old sentences containing one, two, three, and four ideas were $.19,1.31,2.45$, and 3.54 ; corresponding means for new sentences were $-.43,1.09,1.84$, and 2.00 . Trend analyses were performed separately for old and new sentences and in both cases there was a highly significant linear trend $[F(1,52)=26.79$ and 15.84 , respectively].

In order to determine whether the linear trend could be accounted for by frequency measures, regression analyses were performed for the old and new sentences separately. In each analysis the only significant predictor was $F_{1}$. For old sentences $r=.716, F(1,50)=$ 52.49 ; for new sentences $\mathrm{r}=.690, \mathrm{~F}(1,50)=45.36$.

\section{GENERAL DISCUSSION}

In two experiments, $F_{1}$, the frequency of presentation of all simple ideas in a recognition sentence, was shown to be a highly significant predictor of mean recognition ratings. Thus, the suggestion of several authors (Neumann, 1974; Reitman \& Bower, 1973; Underwood \& Zimmerman, 1973) that the linear effect reported by Bransford and Franks (1971) can be accounted for by some form of frequency model is clearly supported by the results of this study. The linguistic integration effect has been widely accepted as a demonstration of the ability of subjects to abstract and integrate information from isolated sentences. However, since the frequency models do not assume that an abstracted representation of the concept or complex idea has been stored in memory, presence of the linear effect is not sufficient to demonstrate the existence of such a representation.

Acquisition frequency appears to be an important determiner of recognition responses, especially when the items presented during acquisition have elements in common and when new items presented during recognition are constructed from those elements. The importance of frequency in studies of word recognition has been demonstrated by Underwood and Zimmerman (1973), and the present study shows that acquisition frequency may affect sentence recognition as well. If this is so, then paradigms which use false recognition as a basis for inferring the nature of memory representations must care- 
fully control for the effects of acquisition frequency to insure that phenomena presumed to reflect encoding in memory are not simply artifacts of decision processes occurring during recognition.

\section{REFERENCES}

Bransford, J. D., \& Franks, J. J. The abstraction of linguistic ideas. Cognitive Psychology, 1971, 2, 331-350.

Cofer, C. N. Constructive processes in memory. American Scientist, 1973, 61, 537-543.

Franks, J. J., \& BRANSFORD, J. D. Abstraction of visual patterns. Journal of Experimental Psychology, 1971, 90, 65-74.

Franks, J. J., \& Bransford, J. D. The acquisition of abstract ideas. Journal of Verbal Learning and Verbal Behavior, 1972, 11, 311-315.

Griggs, R. A. The recall of linguistic ideas. Journal of Experimental Psychology, 1974, 103, 807-809.

KATz, S. Role of instructions in abstraction of linguistic ideas. Journal of Experimental Psychology, 1973, 98, 79-84.

Katz, S., Atkeson, B., \& LEe, J. The Bransford-Franks linear effect: Integration or artifact? Memory \& Cognition, 1974, 2, 709-713.
Katz, S., \& Gruenwald, P. The abstraction of linguistic ideas in "meaningless" sentences. Memory \& Cognition, 1974, 2, 737-741.

NeumanN, P. G. An attribute frequency model for the abstraction of prototypes. Memory \& Cognition, 1974, 2, 241-248.

Reitman, J. S., \& Bower, G. H. Storage and later recognition of exemplars of concepts. Cognitive Psychology, 1973, 4, 194-206.

Singer, M. A replication of Bransford and Franks' (1971) "The abstraction of linguistic ideas". Bulletin of the Psychonomic Society, 1973, 1, 416-418.

Singer, M., \& RosenberG, S. T. The role of grammatical relations in the abstraction of linguistic ideas. Journal of Verbal Learning and Verbal Behavior, 1973, 12, 273-284.

Small, D. W. The abstraction of arbitrary categories. Memory \& Cognition, 1975, 3, 581-585.

UNDERWOOD, B. J. Word recognition memory and frequency information. Journal of Experimental Psychology, 1972, 94, 276-283.

UNDERWOOD, B. J., \& Zimmerman, J. The syllable as a source of error in multisyllable word recognition. Journal of Verbal Learning and Verbal Behavior, 1973, 12, 701-706.

(Received for publication December 4, 1976.) 